\title{
Vehicle Efficiency Maintaining System
}

\author{
Rohit Kothawale ${ }^{1}$, Aniket Mahajan², Prof. Sarika Patil ${ }^{3}$, Prof. Manjusha Namewar ${ }^{4}$ \\ Student, Electronics \& Telecommunication, Sinhgad College of \\ Engineering, Pune, India ${ }^{1}$ \\ Student, Mechanical, Vellore Institute of Technology, Chennai, India ${ }^{2}$ \\ Professor, Electronics \& Telecommunication, Sinhgad College of Engineering, Pune, \\ India ${ }^{3,4}$
}

D.O.I-10.51201/12482

https://doi.org/10.51201/12482

\begin{abstract}
:
This project is based on increasing the fuel efficiency of vehicle. Every vehicle is given an economy speed range by the respective company. But this range will vary due to parameters like the weight of the vehicles and passengers, condition of road, slope of the road, temperature, etc. During real time this economy range will vary. This system will help the driver to maintain actual economy speed. While driving in mountain areas on the slopes, the economy speed range will be different than on plane roads. This system will take all these parameters in account and navigate the driver to get the maximum efficiency in all situations. In addition to this the driver will provide the driver the approximate kilometers the vehicle can travel in the current fuel. The circuit, working and need of the system is briefly discussed in this paper.
\end{abstract}

Keywords: Microcontroller, LCD, Fuel Level Sensor, Gyro Sensor, Ultrasonic Sensor, LED, etc.

\section{INTRODUCTION}

Though the automotive companies provide us with a range of economy speed, they does not take into account the real time data and conditions in which the vehicles are driven. It's an approximate value calculated using the average of all the parameters involved, which cause a significant change in fuel efficiency. We have developed a system which gives us the economy speed of the vehicle using real time conditions i.e. at the time the vehicle is driven, which will be helpful in increasing the fuel efficiency of the vehicle. Our system takes the real time data while driving and calculate the economy speed every 100 meters. The data consists of volume of the fuel measured using a ultrasonic sensor and gyroscope as well as the speed of the vehicle recorded every 100 meters. The data collected will be processed and an economy speed range will be calculated for that distance. Accordingly, the driver will be instructed to increase or decrease the speed in order to match the economy speed at the time. Thus, maximum efficiency can be obtained using this system.

\section{PROPOSED SYSTEM}

Below shown is the block diagram representation of the "VEHICLE EFFICIENCY MAINTAINING SYSTEM". 


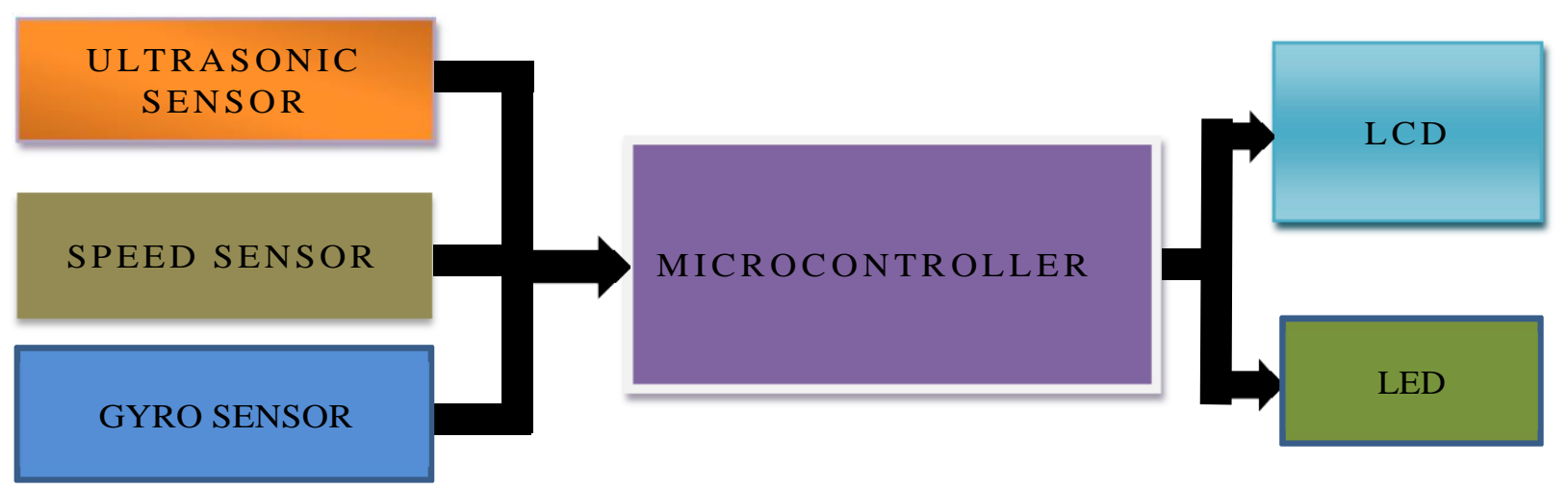

The system consists of total six blocks, the function of the six blocks are as follows:

1. Microcontroller : A microcontroller is a compact integrated circuit designed to govern a specific operation in an embedded system. In this system we are using ARDUINO UNO as microcontroller. The purpose of choosing this as a microcontroller is due to its compact size and number of input/output pins available ( 14 digital and 6 analog pins available) which will make future development of the system of the easy and compact alongside.

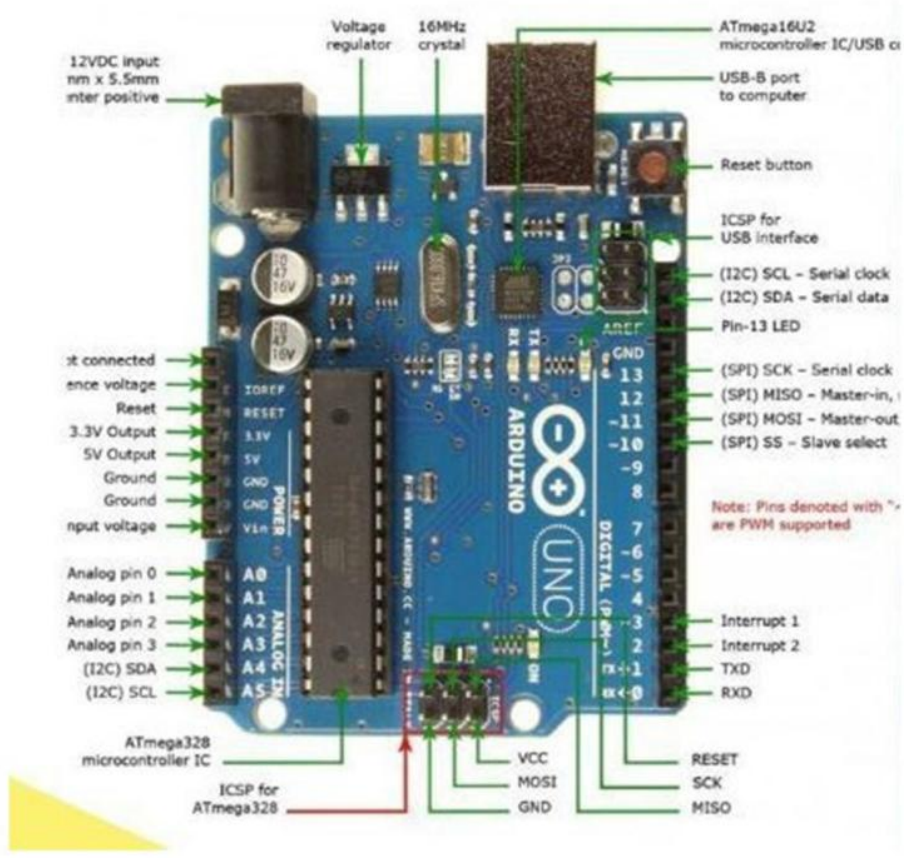

2. Ultrasonic Sensor: An ultrasonic sensor is an electronic device that measures the distance of a target object by emitting ultrasonic sound waves, and converts the reflected sound into an electrical signal. Ultrasonic waves travel faster than the speed of audible sound (i.e. the sound that humans can hear). In this system the purpose of the sensor is to detect the presence of any object within 50 meters of range and send the corresponding signal to the microcontroller. Below shown is a working diagram of an ultrasonic sensor. 


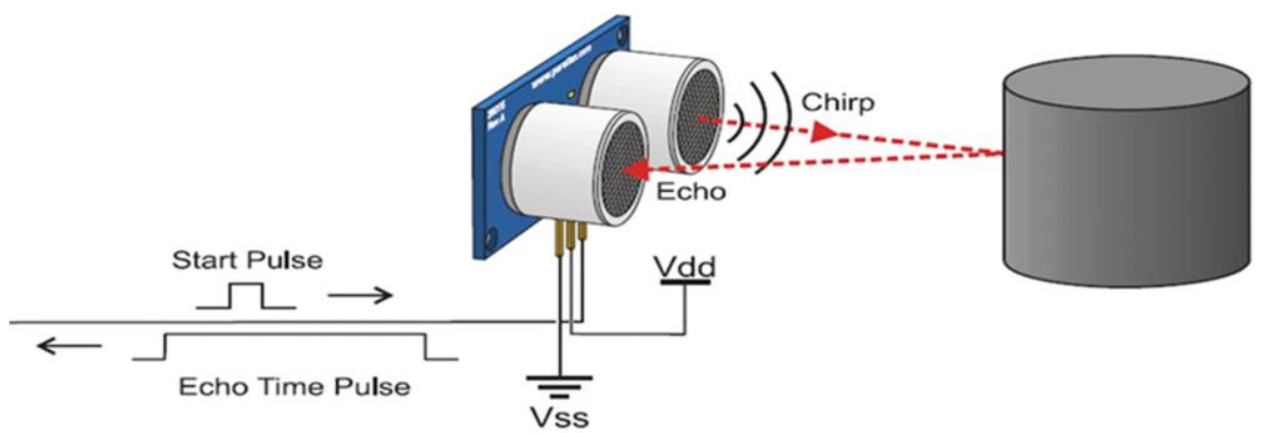

3. LED: LED (Light Emitting Diode) is basically a small light emitting device that comes under "active" semiconductor electronic components. It's quite comparable to the normal general purpose diode, with the only big difference being its capability to emit light in different colors. The two terminals (anode and cathode) of a LED when connected to a voltage source in the correct polarity, may produce lights of different colors, as per the semiconductor substance used inside it.

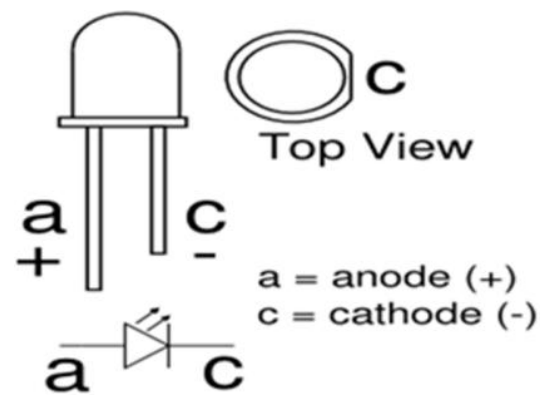

4. Speed Sensor (LM393): The LM393 Speed Sensor Module is basically an Infrared Light Sensor integrated with LM393 Voltage Comparator IC..The sensor part of the LM393 Speed Sensor module consists of an Infrared LED and an NPN Photo Transistor. These two components are placed facing each other is a special housing made of black thermoplastic.

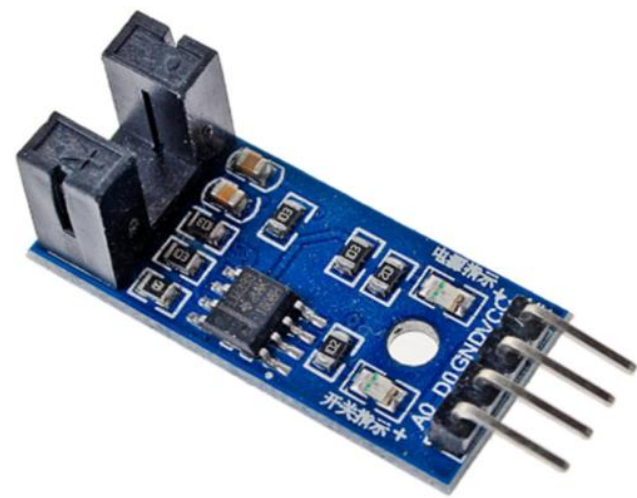


5. LCD: The term LCD stands for liquid crystal display. It is one kind of electronic display module used in an extensive range of applications like various circuits \& devices like mobile phones, calculators, computers, TV sets, etc. LCD modules are very commonly used in most embedded projects, the reason being its cheap price, availability and programmer friendly. LCD is presented as dashboard of the vehicle which will show us the speed, fuel level, remaining kilometers ,etc.

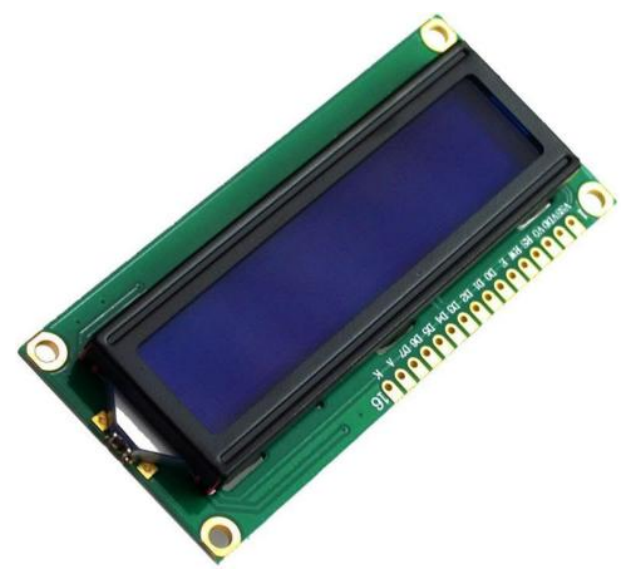

6. Gyro Sensor: Gyro sensors, also known as angular rate sensors or angular velocity sensors, are devices that sense angular velocity. Angular velocity. In simple terms, angular velocity is the change in rotational angle per unit of time. Angular velocity is generally expressed in degree/s (degrees per second).

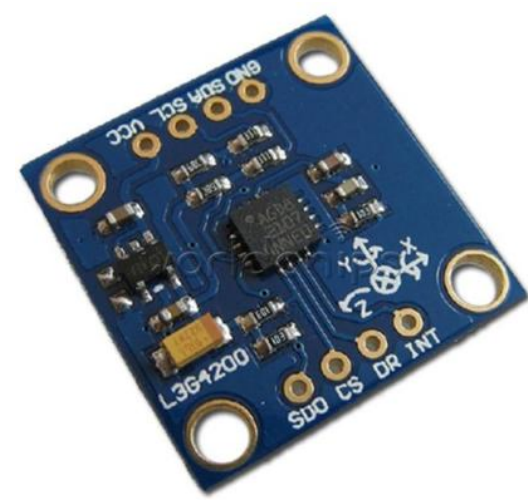

\section{NEED OF SYSTEM}

Fuel efficiency of a vehicle varies due to many factors, such as:

- Maintaining an efficient speed is an important factor in fuel efficiency. Optimal efficiency can be expected while cruising at a steady speed, at minimal throttle and with the transmission in the highest gear. The optimal speed varies with the type of vehicle, although it is usually reported to be between $35 \mathrm{mph}$ $(56 \mathrm{~km} / \mathrm{h})$ and $50 \mathrm{mph}(80 \mathrm{~km} / \mathrm{h})$. For instance, a 2004 Chevrolet Impala had an optimum at $42 \mathrm{mph}(70 \mathrm{~km} / \mathrm{h})$, and was within $15 \%$ of that from 29 to 57 $\mathrm{mph}$ (45 to $95 \mathrm{~km} / \mathrm{h}$ ). At higher speeds, wind resistance plays an increasing role in reducing energy efficiency. 
- Road capacity affects speed and therefore fuel efficiency as well. Studies have shown speeds just above $45 \mathrm{mph}(72 \mathrm{~km} / \mathrm{h})$ allow greatest throughput when roads are congested. Individual drivers can improve their fuel efficiency and that of others by avoiding roads and times where traffic slows to below $45 \mathrm{mph}$ $(72 \mathrm{~km} / \mathrm{h})$. Communities can improve fuel efficiency by adopting speed limits or policies to prevent or discourage drivers from entering traffic that is approaching the point where speeds are slowed below $45 \mathrm{mph}(72 \mathrm{~km} / \mathrm{h})$. Congestion pricing is based on this principle; it raises the price of road access at times of higher usage, to prevent cars from entering traffic and lowering speeds below efficient levels.

$\square \quad$ Engine efficiency varies with speed and torque. For driving at a steady speed one cannot choose any operating point for the engine-rather there is a specific amount of power needed to maintain the chosen speed. A manual transmission lets the driver choose between several points along the power band. For a turbo diesel too low a gear will move the engine into a high-rpm, low-torque region in which the efficiency drops off rapidly, and thus best efficiency is achieved near the higher gear. In a gasoline engine, efficiency typically drops off more rapidly than in a diesel because of throttling losses. Because cruising at an efficient speed uses much less than the maximum power of the engine, the optimum operating point for cruising at low power is typically at very low engine speed, around or below $1000 \mathrm{rpm}$. This explains the usefulness of very high "overdrive" gears for highway cruising. For instance, a small car might need only $10-15$ horsepower $(7.5-11.2 \mathrm{~kW})$ to cruise at $60 \mathrm{mph}(97 \mathrm{~km} / \mathrm{h})$. It is likely to be geared for $2500 \mathrm{rpm}$ or so at that speed, yet for maximum efficiency the engine should be running at about $1000 \mathrm{rpm}$ to generate that power as efficiently as possible for that engine (although the actual figures will vary by engine and vehicle).

- Fuel efficiency varies with the vehicle. Fuel efficiency during acceleration generally improves as RPM increases until a point somewhere near peak torque (brake specific fuel consumption). However, accelerating to a greater than necessary speed without paying attention to what is ahead may require braking and then after that, additional acceleration. Experts recommend accelerating quickly, but smoothly.

- Generally, fuel efficiency is maximized when acceleration and braking are minimized. So a fuel-efficient strategy is to anticipate what is happening ahead, and drive in such a way so as to minimize acceleration and braking, and maximize coasting time.

$\square \quad$ The need to brake is sometimes caused by unpredictable events. At higher speeds, there is less time to allow vehicles to slow down by coasting. Kinetic energy is higher, so more energy is lost in braking. At medium speeds, the driver has more time to choose whether to accelerate, coast or decelerate in order to maximize overall fuel efficiency. 
- While approaching a red signal, drivers may choose to "time a traffic light" by easing off the throttle before the signal. By allowing their vehicle to slow down early and coast, they will give time for the light to turn green before they arrive, preventing energy loss from having to stop. Due to stop and go traffic, driving during rush hours is fuel inefficient and produces more toxic fumes.

$\square$ Conventional brakes dissipate kinetic energy as heat, which is irrecoverable. Regenerative braking, used by hybrid/electric vehicles, recovers some of the kinetic energy, but some energy is lost in the conversion, and the braking power is limited by the battery's maximum charge rate and efficiency.

$\square \quad$ Using high octane gasoline fuel in a vehicle that does not need it is generally considered an unnecessary expense, although Toyota has measured slight differences in efficiency due to octane number even when knock is not an issue. If the engine is designed for high octane then higher octane fuel will result in higher efficiency and performance under certain load and mixture conditions. The energy released during combustion of hydrocarbon fuel increases as the molecule chain length decreases, so gasoline fuels with higher ratios of the shorter chain alkanes such as heptane, hexane, pentane, etc. can be used under certain load conditions and combustion chamber geometries to increase engine output which can lead to lower fuel consumption, although these fuels will be more susceptible to pre detonation ping in high compression ratio engines. Gasoline direct injection compression ignition engines make more efficient use of the higher combustion energy short chain hydrocarbons as the fuel is injected directly into the combustion chamber during high compression which auto-ignites the fuel, minimizing the amount of time that the fuel is available in the combustion chamber for pre detonation.

Fuel efficiency is a form of thermal efficiency, meaning the ratio of effort to result of a process that converts chemical potential energy contained in a carrier (fuel) into kinetic energy or work. Overall fuel efficiency may vary per device, which in turn may vary per application. During present time, a speed ranging between of 40-60 kilo meters per hour is considered to be an ideal conditional to drive a vehicle, also called as "Economy Speed". Driving the vehicle in this particular speed range ensures us about minimum fuel usage and maximum output. This is basically a generalized speed range calculated using the average of all the parameters which affect the fuel efficiency of a vehicle. It does not take into account the actual conditions in which the vehicle is driven at present.

On the contrary, the system which we have developed uses real time data to calculate the economy speed of the vehicle and instructs the driver whether to increase or decrease the speed in order to achieve the economy speed at that point in time and hence, increases the fuel efficiency of the vehicle. Our system takes into account the real time data consisting of the speed of the vehicle, the volume of the fuel in the tank(using ultrasonic sensor and gyroscope). 


\section{WORKING}

There are two applications of this project.

- The first application of this project is that the exact remaining kilometres the vehicle can travel with the fuel is shown on the dashboard. The Ultrasonic readings are given as input to the microcontroller.

$$
\text { Distance }=\frac{\text { Time } x \text { Speed of Sound in Air }(343 \mathrm{~m} / \mathrm{s})}{2}
$$

The location of the Ultrasonic Sensor placed is shown below.

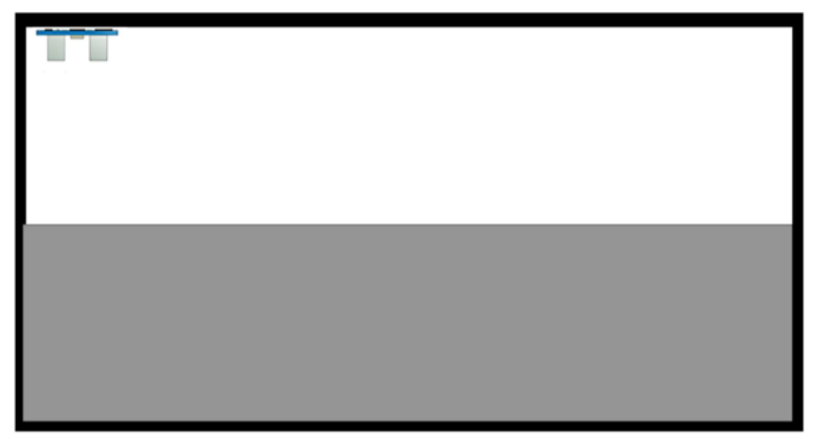

The Ultrasonic Sensor will give the fuel level data to the microcontroller. But there may be situation where the vehicle will be on slope and due to the tilting of the fuel tank the data received will be including order to get accurate data in these situations, there is one more parameter considered that is the angle of tilting. For this ,Gyro Sensor is used. Gyro Sensor will give the angular motion of the vehicle. When the vehicle is travelling on plane road, only the Ultrasonic readings will be considered as the fuel level. But while driving on slope, the fuel tank will get tilted will give incorrect fuel readings. So the Gyro sensor and Ultrasonic readings will be processed and we will get the exact fuel level.

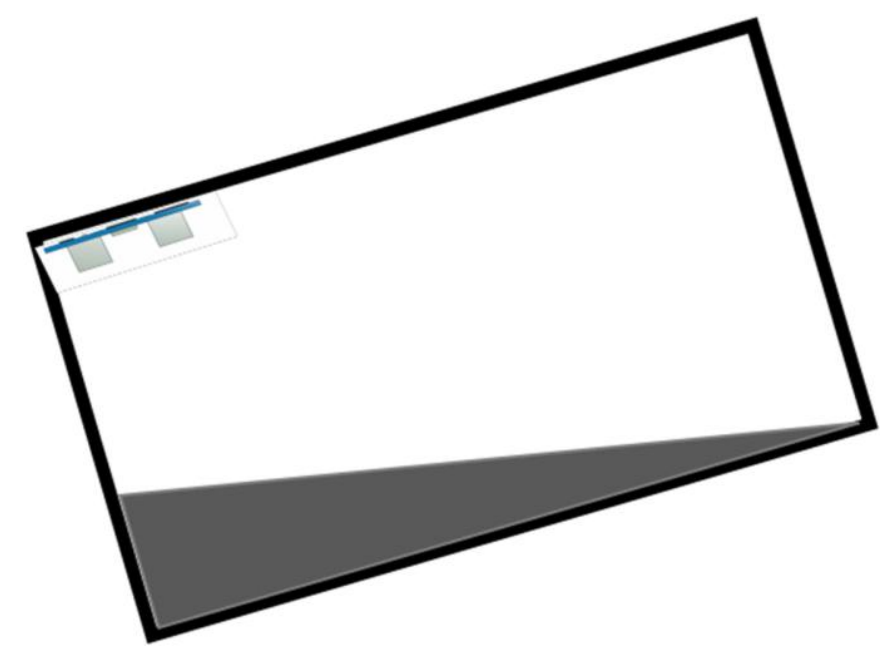


- The fuel data will be converted in percentage out of total fuel that can be accommodated in the fuel tank. This percentage data will be given as output to the dashboard of the car.

- The Speed Sensor readings will be given as input to the microcontroller. After every 25 meters, the speed sensor readings data will be taken. After 100 meter the amount of fuel reduced will be processed. This will give us the data of kilometres the vehicle can travel with the fuel left. This process will give the driver the exact kilometres the vehicle will travel with the present fuel. This data will vary according to the speed of vehicle, the temperature of the engine and change in roads. The data of the kilometres left will be refreshed after every 100 metres .

- The next application of this project is to help the driver to increase the efficiency of the vehicle. The system will suggest the driver to vary the speed of the vehicle accordingly to get the maximum efficiency. After every 25 meters, the speed of the vehicle is given to the microcontroller. The microcontroller will stack this data . Four speed readings will be compared and average of them will be considered. After every 25 meters, the first one of the four readings will be cleared and new speed reading will be stored in the stack . Due to this , the real time mileage of the vehicle will be obtained. This mileage will be used to find the exact kilometres the vehicle will travel with the remaining fuel. This exact kilometres data will be shown on dashboard of the vehicle which will help the driver to know when to refill the fuel tank.

- The mileage and the present speed readings will be processed . After every 25 meters the mileage data will be stacked and after four readings the data will be compared and we will get the efficient speed at which the vehicle should be driven at that time. The system will navigate the driver to maintain the speed to get maximum the efficiency. The microcontroller will give input to the dashboard which will tell the driver to increase or reduce speed. Two LEDS of arrows shape pointing in upward and downward direction. If the driver is exceeding than present economy speed, the microcontroller will turn on the arrow pointing downward which will indicate the driver to reduce the speed and vice a versa.
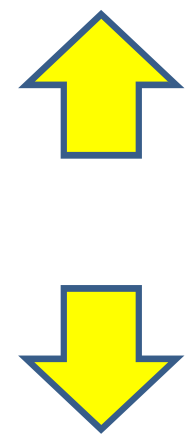


\section{CIRCUIT DIAGRAM}

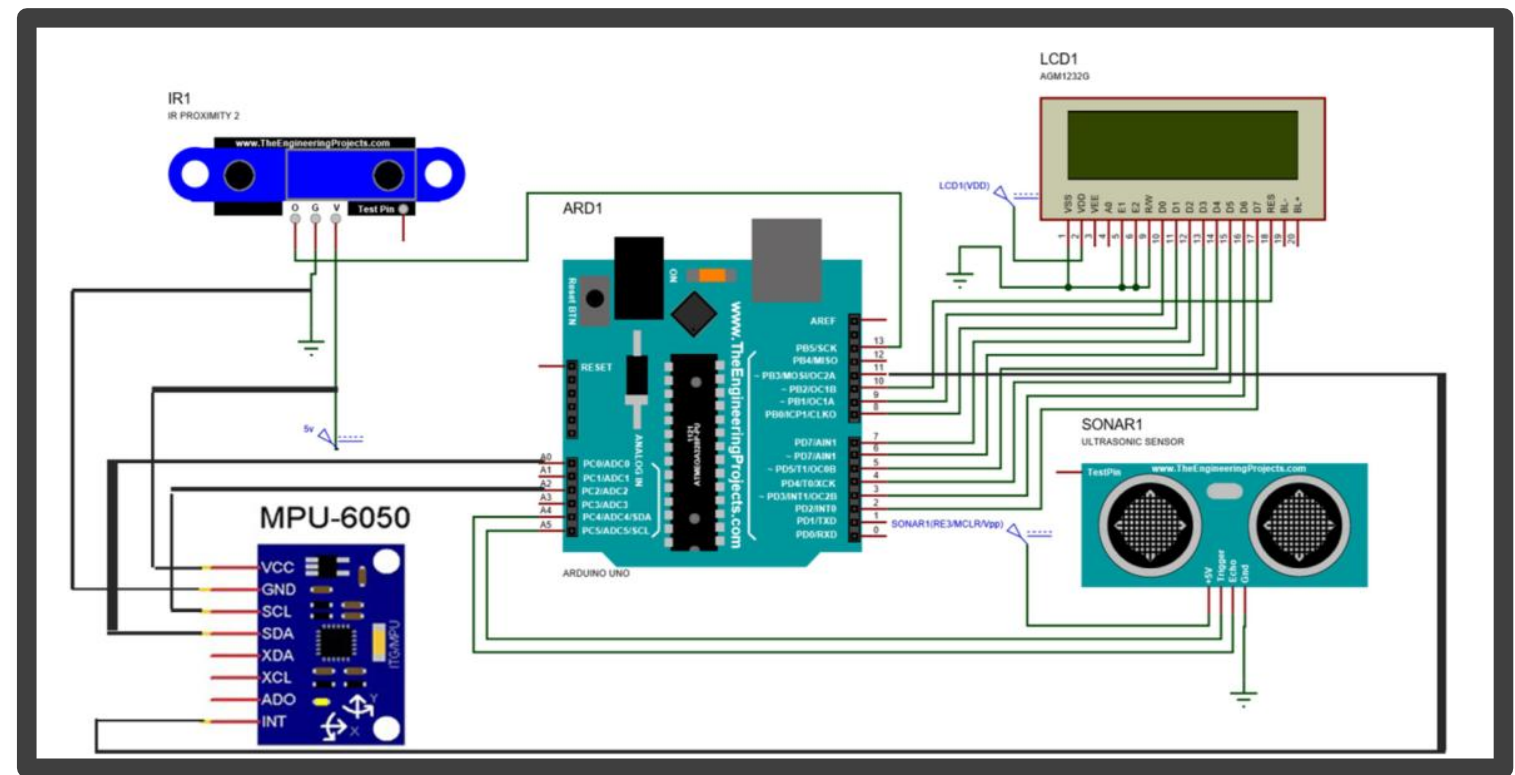

\section{REFERENCES}

[1]. https://www.azosensors.com/article.aspx?ArticleID=1616

[2]. https://users.encs.concordia.ca/ bwgordon/arduino_lab3.html

[3]. https://www.elprocus.com/lcd-16x2-pin-configuration-and-its-working/

[4]. https://www.electronicshub.org/interfacing-lm393-speed-sensor-with-arduino/

[5]. https://components101.com/microcontrollers/arduino-uno

[6]. https://en.wikipedia.org/wiki/Energy-efficient_driving

[7]. https://www.arduino.cc/en/Main/software

[8]. https://www.elprocus.com/gyroscope-sensor/

[9]. https://en.wikipedia.org/wiki/Soil_moisture_sensor 